BNL-61260

\title{
SPIN-DENSITY-WAVE MAGNETISM IN DILUTE COPPER-MANGANESE ALLOYS
}

F. J. LAMELAS," S. A. WERNER, ${ }^{* *}$ S. M. SHAPIRO, ${ }^{* *}$ and J. A. MYDOSH****

*Department of Physics, Marquette University, Milwaukee, WI 53233

**Department of Physics, The University of Missouri, Columbia, Missouri 65211

*** Department of Physics, Brookhaven National Laboratory, Upton, New York 11973

****Kammerlingh Onnes Laboratory, Rijksuniversiteit, 2300 RA Leiden, The Netherlands

\section{ABSTRACT}

Elastic neutron-scattering measurements on two samples of $\mathrm{Cu}$ alloyed with $1.3 \% \mathrm{Mn}$ and $0.55 \% \mathrm{Mn}$ show that the spin-density-wave (SDW) features found in more concentrated alloys persist in the limit of very dilute alloys. These features consist of temperature-dependent incommensurate peaks in magnetic neutron scattering, with positions and strengths which are fully consistent with those in the concentrated alloys. The implications of these measurements are twofold. First, it is clear from our data that SDW magnetic ordering occurs across the entire range of $\mathrm{CuMn}$ alloys which have typically been interpreted as spin glasses. Second, the more fundamental significance of this work is the suggestion via extrapolation that a peak in the magnetic susceptibility $\chi(\mathbf{q})$ occurs in pure copper, at a value of $q$ given by the Fermi-surface diameter $2 k_{F}$.

\section{INTRODUCTION}

In condensed-matter physics, structural measurements can be considered fundamental in that all theories and experiments must be consistent with the structural data. For magnetic materials, the most powerful structural probe available is neutron scattering. However, in the study of $\mathrm{CuMn}$ spin-glasses, crucial neutron scattering results ${ }^{1}$ were obtained rather late in the development of the field ${ }^{2}$ and thus their impact has been limited. This situation has arisen because of the relative weakness of the scattering associated with spin ordering, and also because the interpretation of the neutron data has evolved slowly over the past decade, aided, to a significant degree, by new theoretical developments. ${ }^{3,4}$ In this paper we discuss a $\mathrm{Cu}-0.55 \% \mathrm{Mn}$ crystal which represents a probable limit in the composition which is accessible with current neutron scattering sources and techniques. The current data, together with earlier measurements on more concentrated alloys, bracket the range of compositions which has formed the subject of intensive spin-glass studies.

The neutron data form a picture which is rather at odds with the standard view ${ }^{5}$ of the nature of a spin glass. Taken as a whole, the scattering measurements suggest that the most straightforward interpretation of CuMn alloys is that of a spin-density-wave (SDW) antiferromagnet (AFM). In the SDW picture, the spins of the valence electrons are polarized antiferromagnetically, with a wave vector which is incommensurate with the reciprocal lattice. ${ }^{6}$ This is in contrast to the case of an ordinary AFM, where the magnetic lattice is commensurate with the structural lattice. As discussed below, the incommensurate nature of the SDW is due to its origin as a Fermi-surface effect. Although the SDW arises due to the properties of the copper-valence-electron gas, it results in the spin polarization of

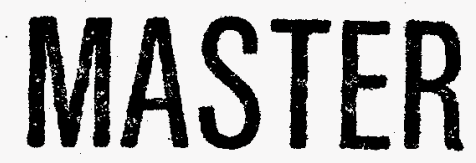


the manganese impurity ions which are more-or-less randomly situated within the copper matrix. Experimentally, the SDW is detected as a distinct neutron-scattering peak, with a scattering intensity proportional to the square of the manganese-ion concentration.

One would expect that SDW domain sizes would vary with sample preparation, and that consequently different neutron-scattering measurements would reveal SDW peaks of varying widths. However the $\mathrm{CuMn}$ experiments have uniformly led to the observation of SDW peak widths corresponding to domains which are only ten FCC unit cells in extent. That is, the SDW does not develop into a state of long-range order. We believe that the solution to this puzzle reflects the intrinsic nature of antiferromagnetism in $\mathrm{Cu}$ alloys. It can be explained by SDW ordering which arises due to a peak in the wave-vector-dependent magnetic susceptibility $\chi(\mathbf{q})$. If the peak in $\chi(\mathbf{q})$ is relatively weak and broad, the ordering of the Mn ions (detected by the neutron-scattering measurements) will be short-ranged, leading to peak widths which are broadened not due to sample preparation artifacts, but rather as a direct manifestation of the shape of $\chi(\mathbf{q}){ }^{4}{ }^{4}$ Thus the neutron-scattering data for dilute alloys serve as a probe of the wave-vector-dependent magnetic susceptibility of pure copper metal.

\section{EXPERIMENTAL DETAILS}

Our measurements were performed on samples prepared by standard methods in Leiden. The first crystal, with a composition of $\mathrm{Cu}-1.3$ at.\% $\mathrm{Mn} \pm 0.2 \%$ was grown by single-pass zone melting in a graphite crucible under Ar. A second sample, of composition $\mathrm{Cu}-0.55$ at.\% $\mathrm{Mn} \pm 0.07 \%$ was prepared using the Czochralski technique. This crystal was annealed at $950{ }^{\circ} \mathrm{C}$ to remove strains and promote homogeneity. The mosaics of the samples were $1.2^{\circ}$ for $1.3 \% \mathrm{Mn}$ and $0.6^{\circ}$ for $0.55 \% \mathrm{Mn}$. The samples were cylindrical, approximately $50 \mathrm{~mm}$ long and $12 \mathrm{~mm}$ in diameter. Neutron scattering measurements were carried out on triple-axis spectrometer $\mathrm{H} 7$ at the High-Flux Beam Reactor (HFBR) at Brookhaven National Laboratory (BNL). Using a pyrolytic graphite (PG) 002 monochromator and analyzer with a mosaic of $24^{\prime}$, the neutron energy was set to $14.8 \mathrm{meV}(\lambda=2.35 \AA)$. The spectrometer collimation was set at $40^{\prime}-40^{\prime}-40^{\prime}-80^{\prime}$. This yields an instrumental resolution function with a full width at half maximum (FWHM) of 0.04 reciprocal lattice units (RLU) in the scans discussed here, where $1 \mathrm{RLU}=2 \pi / a$ and $a=3.61 \AA$. The energy resolution in this configuration (FWHM) is $0.8 \mathrm{meV}$. In addition, a $50 \mathrm{~mm}$-thick PG filter was used between the sample and analyzer in order to attenuate higher-order neutrons passed by the monochromator. To access a wide range of temperatures, the samples were mounted in a pumped He flow cryostat with a base temperature of $1.4 \mathrm{~K}$.

\section{RESULTS}

Twenty-five years ago Krivoglaz ${ }^{7}$ and Moss $^{8}$ pointed out that Fermi-surface effects in the ordering of metallic alloys can be seen in x-ray scattering experiments. In this case, an enhancement in the solvent-solute potential $V(\mathbf{q})$ can occur for values of $\mathbf{q}$ which span the Fermi surface, that is, when $\mathbf{q} \simeq 2 k_{F}$. If one draws a surface of radius $2 k_{F}$ about each of the reciprocal-lattice points of the solvent metal, the effect is accentuated at the crossings of adjacent surfaces. Such a feature in the potential results in solute ordering with this wave vector, which in turn leads to peaks in X-ray scattering at the Fermi-surface crossings generated by the construction described above. In $\mathrm{CuMn}$, the observed AFM 


\section{DISCLAIMER}

Portions of this document may be illegible in electronic image products. Images are produced from the best available original document. 


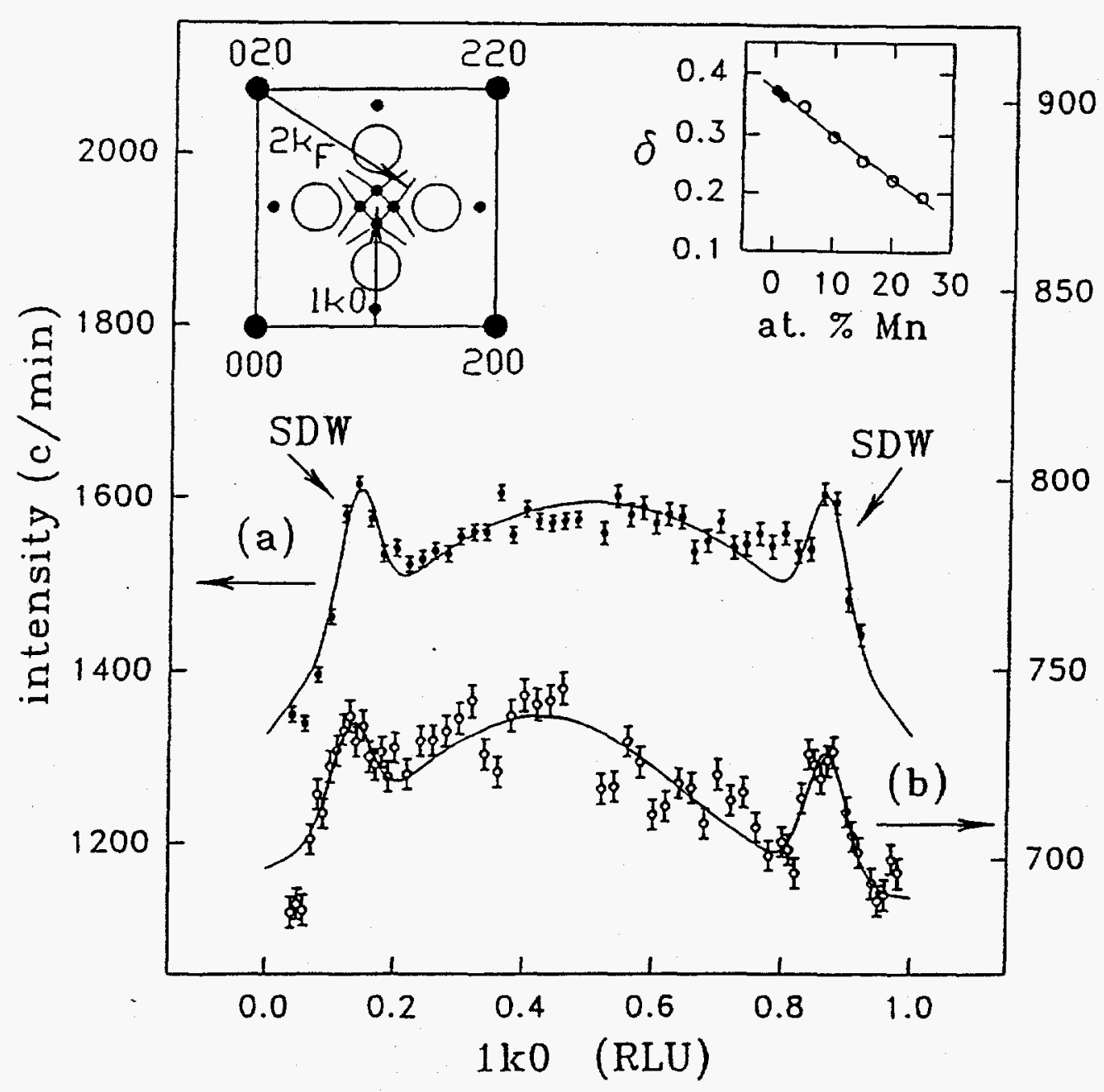

Figure 1. Elastic neutron scattering intensities along $1 \mathrm{k} 0$ at $\mathrm{T}=1.4 \mathrm{~K}$ for (a) $\mathrm{Cu}-1.3 \%$ $\mathrm{Mn}$ and (b) $\mathrm{Cu}-0.55 \% \mathrm{Mn}$. The counting times per point were 18 minutes for the $1.3 \% \mathrm{Mn}$ sample and 44 minutes for the $0.55 \% \mathrm{Mn}$ sample. In the inset at the upper left, reciprocal-space positions of incommensurate SDW peaks are shown as small filled circles; commensurate ASRO peaks are shown as large open circles. The $1 \mathrm{k} 0$ scan trajectory is shown by a vertical arrow, and the crossed lines in the center of the inset illustrate the Fermi-surface construction described in the text. In the inset at the upper right, incommensurate SDW peak positions are plotted against Mn composition. The incommensurability parameter $\delta$ is defined as 0.5 minus the k-value of the first peak shown in the scan trajectory. In RLU, $4 \mathrm{k}_{\mathrm{F}}{ }^{2}=1^{2}+(1.5-\delta)^{2}$. Data from Ref. 10 are shown as open circles, while the new data are shown as filled circles. 
neutron-scattering peaks occur at precisely such crossings (Fig. 1, left inset), thus the first thing that one should note is that the peak positions are unambiguous indicators that the magnetic ordering is due to a Fermi-surface effect. In concentrated CuMn alloys the scattering intensities are high enough to allow a mapping of the intensities over the $h k 0$ plane. ${ }^{1}$ For the dilute alloys discussed here, we discuss scans along $1 k 0$, as indicated in Fig. 1 .

Fig. 1 shows that SDW peaks persist in CuMn alloys containing as little as $0.55 \%$ $\mathrm{Mn}$. The solid curves in this figure were generated by fitting the measured data to three Gaussians plus a background. In addition, the central Gaussian for the $0.55 \% \mathrm{Mn}$ sample was multiplied by a linear function, in order to duplicate the asymmetric shape of the measured peak. The right-hand inset of Fig. 1 shows that SDW peak positions follow those found in more concentrated alloys. The variation of peak position with $\mathrm{Mn}$ concentration is an important proof that the neutron scattering peaks are at Fermi-surface crossings: Mn acts as a donor impurity, thus $k_{F}$ increases with Mn concentration and the crossings shift in position.

The temperature dependence of individual SDW features in the two alloys is shown in Fig. 2. The SDW peak intensities obtained by the fitting procedure are shown in Fig. 3 . In addition, the intensity of the broad feature at $k=0.5$ in Fig. 2 was fitted as a function of temperature, in order to generate a temperature-dependent background. Aside from the peak intensities found by fitting entire peak profiles, for the sample containing $1.3 \% \mathrm{Mn}$ peak intensities a a single q-value were measured at additional temperatures and the background function was subtracted. These additional points are included in Fig. 3.

If the macroscopic magnetic properties in CuMn arise from SDW ordering, then the SDW scattering intensity should act as an order parameter and drop to zero near the spinglass freezing temperature $T_{f}$. Based on magnetic susceptibility measurements, ${ }^{9} T_{f} \sim 6.5$ $\mathrm{K}$ at $0.55 \% \mathrm{Mn}$ and $T_{f} \sim 14 \mathrm{~K}$ at $1.3 \% \mathrm{Mn}$. However, Fig. 3 shows a slow drop-off in SDW peak intensities as a function of temperature. As discussed previously, ${ }^{10,11}$ this effect is explained as follows. Given the instrumental resolution function, in a quasielastic scattering measurement one integrates $S(\mathbf{q}, \omega)$ over a range of $\omega$ on either side of $\omega=0$. If the integrated inelastic scattering is relatively strong in comparison to $S(\mathbf{q}, \mathbf{0})$ and in addition has a weak temperature dependence, the measured quasielastic scattering can remain significant well above the Néel temperature. In CuMn, inelastic neutron-scattering measurements on more concentrated samples have shown that excitations with essentially vertical dispersion relations originate at the incommensurate SDW peak positions. ${ }^{12}$ Moreover, constant-q spectral-distribution scans show that our experimental resolution is such that significant elastic-scattering intensity can be measured at nominal energy shifts of up to $1.5 \mathrm{meV}$ (Fig. 4). These two observations make it quite feasible that in our setup one can measure significant scattering intensities well above $T_{f}$, even when the strictly elastic scattering $S(\mathbf{q}, 0)$ is very small. The test for such a conjecture is to repeat the elastic scans as a function of temperature, after significantly increasing the spectrometer $\omega$ resolution. Unfortunately, such test measurements are essentially impossible to perform on dilute CuMn samples, due to count-rate limitations. Nevertheless, it has been shown in more concentrated $\left(15 \% \mathrm{Mn}^{10}\right.$ and $8 \% \mathrm{Mn}^{11}$ ) samples that increasing the $\omega$ resolution produces a significantly sharper drop in quasielastic magnetic scattering intensities with increasing temperature. 


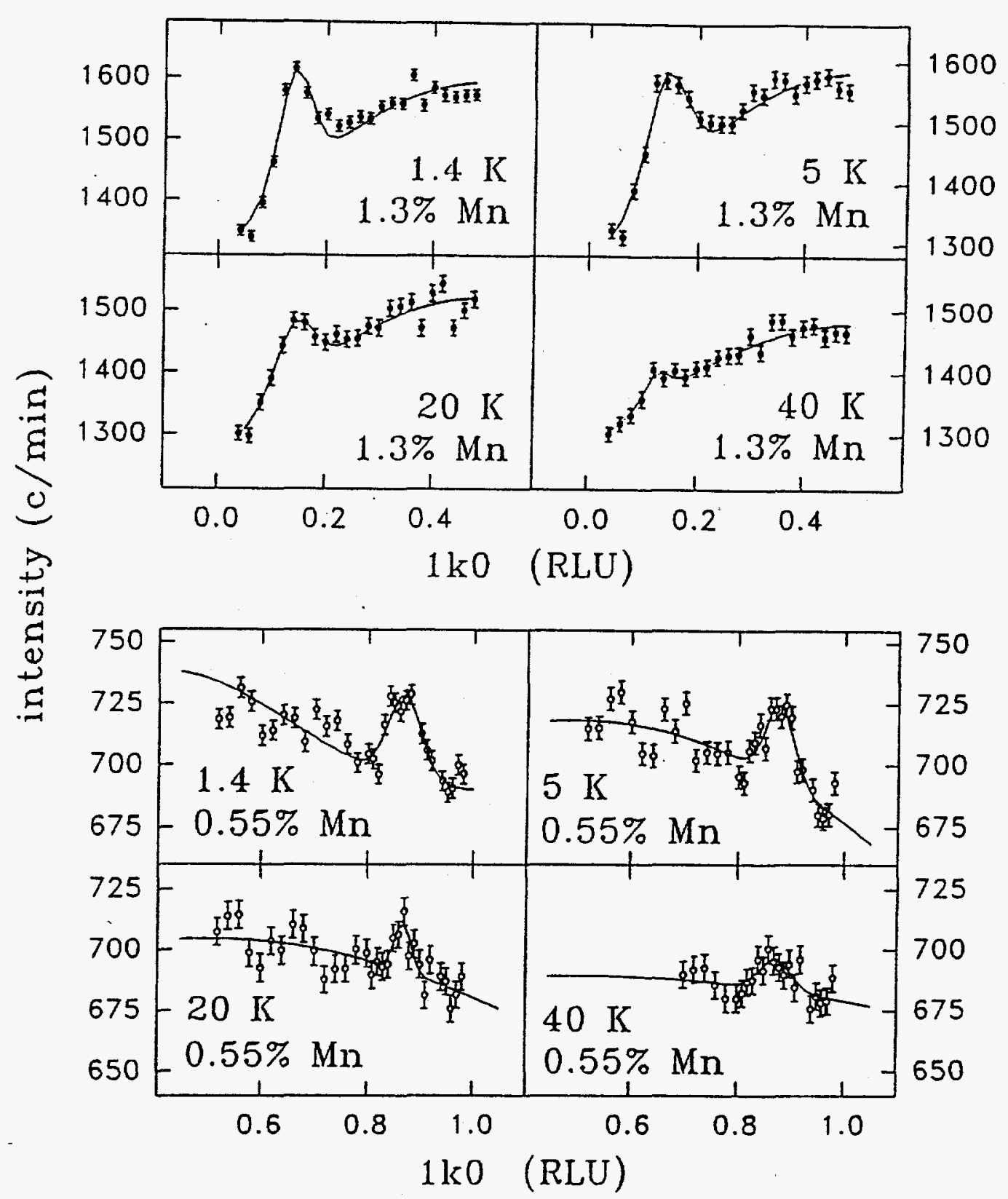

Figure 2. Temperature dependence of the SDW peaks, for $\mathrm{Cu}-1.3 \% \mathrm{Mn}$ (upper plots with filled symbols) and $\mathrm{Cu}-0.55 \% \mathrm{Mn}$ (lower plots, open symbols). For the $1.3 \% \mathrm{Mn}$ sample, the counting times per point were 18 minutes at $\mathrm{T}=1.4 \mathrm{~K}$ and 9 minutes at $\mathrm{T}=5,20$, and $40 \mathrm{~K}$. For the $0.55 \% \mathrm{Mn}$ sample, the counting times were 44 minutes at $1.4 \mathrm{~K}, 33$ minutes at $5 \mathrm{~K}$, and 22 minutes at $\mathrm{T}=20 \mathrm{~K}$ and $40 \mathrm{~K}$. 


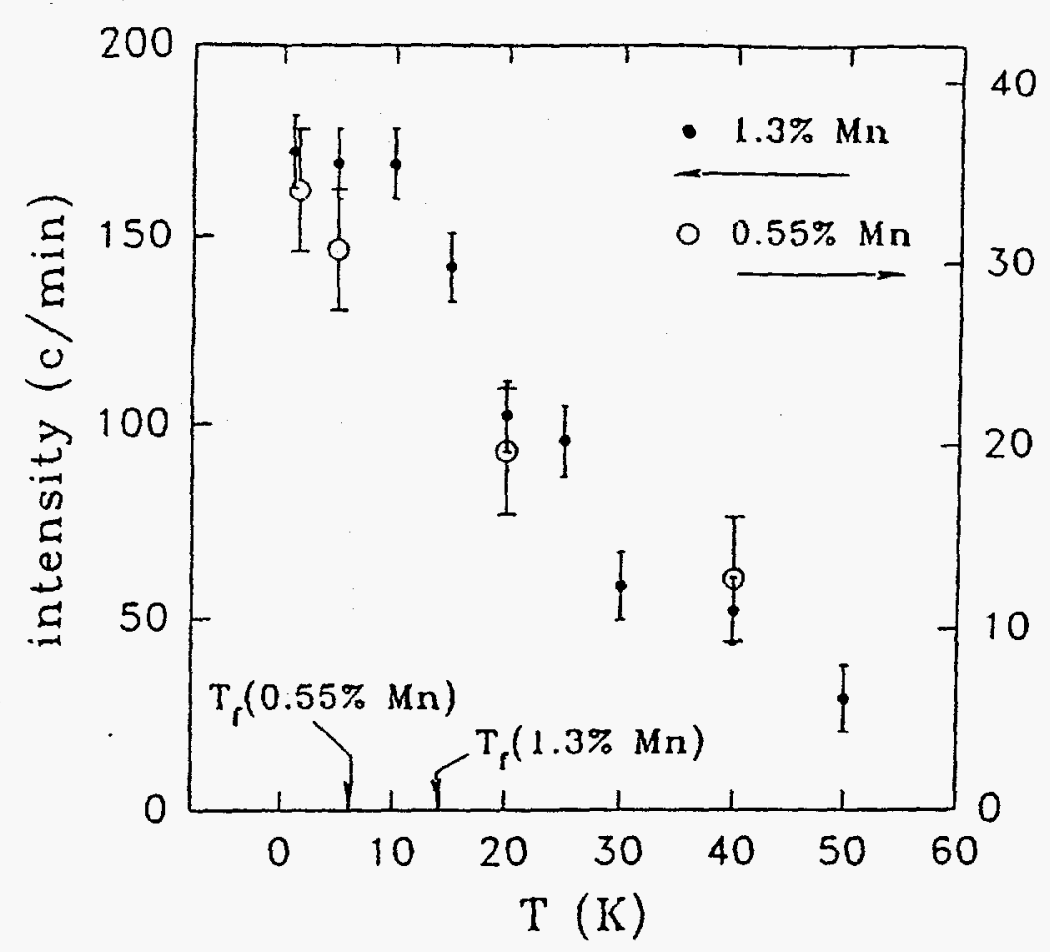

Figure 3. Temperature dependence of the SDW peak intensities for $1.3 \% \mathrm{Mn}$ (filled symbols) and $0.55 \% \mathrm{Mn}$ (open symbols). The intensities used in this plot are primarily derived from Fig. 2, with additional points described in the text.

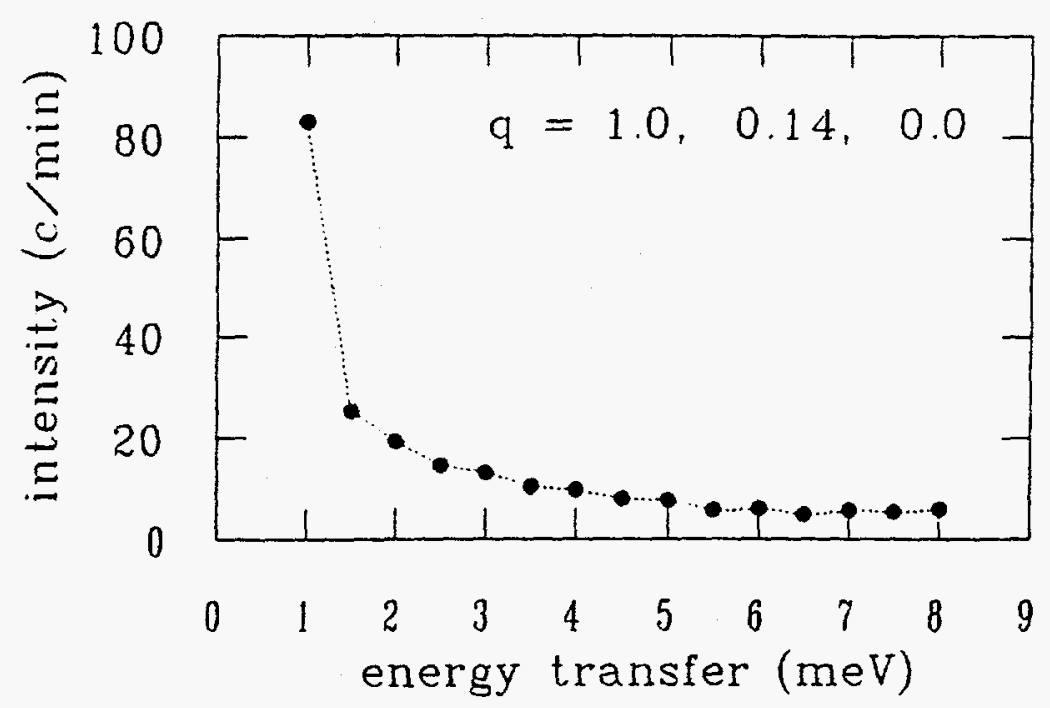

Figure 4. Inelastic constant-q spectral-distribution scan for $\mathrm{Cu}-1.3 \% \mathrm{Mn}$. $\mathrm{T}=1.3 \mathrm{~K}$ and $q=1.0,0.14,0.0$. 


\section{DISCUSSION}

There are a number of details which are common to all of the neutron data taken to date. First, we note that intensities in magnetic scattering are governed by the form factor $f(q)$ of the spin-polarized orbitals. Thus it has not been possible to detect any polarization of $\mathrm{Cu}$ conduction electrons, since their wavefunction is extended and has a form factor which approaches zero starting at very small values of $q$. Magnetism in the Mn ions, on the other hand, arises in the localized $3 \mathrm{~d}$ orbitals, producing a form factor which falls off relatively slowly. Therefore, magnetic scattering in $\mathrm{CuMn}$ alloys serves as a probe of only the Mn spins. It is also clear that the magnetic component of the broad half-integer peak at $k=0.5$ is ferromagnetic, since it coincides in position and width with an atomic-shortrange-order (ASRO) structural peak. (Magnetic peaks due to AFM ordering would occur at reciprocal lattice points corresponding to a unit cell larger than the structural unit cell.) In contrast, spin-polarized neutron scattering measurements showed that the incommensurate SDW peaks arise from AFM scattering and are unrelated to structural ordering. ${ }^{1}$

Another aspect common to all of the neutron data is the nearly linear relation between incommensurate peak position and Mn concentration which was noted above (Fig. 1, right inset). The continuous shift in peak positions shows that the incommensurate peaks are manifestly characteristic of the alloy. That is, the concentration-dependence of the peak positions shows that they are not due to the formation of stable clusters or other chemical ordering phenomena. In that case, the SDW peak positions would asymptotically approach a value corresponding to the cluster, rather than directly following the magnitude of the alloy Fermi wavevector $k_{F}$. This latter type of behavior is in fact found with the ferromagnetic peaks at the half-integer ASRO reciprocal-lattice points: as the Mn concentration is varied their intensities and widths vary, but not their positions. As a final note, it is also significant that the extrapolated value of the SDW incommensurability parameter $\delta$ (Fig. 1) yields a Fermi-surface diameter in precise agreement with de Haas-van Alphen measurements in pure copper. ${ }^{13}$

Before discussing the SDW interpretation of the data, we mention other theories which yield ordering for dilute magnetic systems with short range interactions. Perhaps the simplest such theory is the near-neighbor Ising model worked out originally by Sato et al. ${ }^{14}$ Regardless of the interaction strength, a stable AFM phase results when $c z \geq 2$, where $c$ is the concentration of solute atoms and $z$ is the coordination number. For CuMn, with an exchange coefficient of the appropriate sign, antiferromagnetism would occur at Mn concentrations greater than $16.7 \%$. A similar cutoff occurs with the modified-RKKY-interaction model of Ioffe and Feigel'man. ${ }^{3}$ Here the Mn-Mn potential is of the form $r^{-1} \sin \left(2 k_{F} r\right) e^{-\kappa r}$, which extends beyond near neighbors, bringing the critical concentration of Mn necessary for AFM ordering down to $\sim 10 \%$. It has already been pointed out by Cable and Tsunoda ${ }^{15}$ that this model is inconsistent with the neutron data for dilute CuMn alloys.

Rather than explicitly working with an interaction potential which is written as a function of the Mn-Mn separation, Fajen and Vignale ${ }^{4}$ start with an interaction which takes place via conduction electron polarization:

$$
\mathcal{H}=-\frac{G^{2}}{2 L} \sum_{\mathbf{q}} \chi(\mathbf{q})|\mu(\mathbf{q})|^{2},
$$

where $\mu(\mathbf{q})$ is the Fourier transform of the distribution of the $L \mathrm{Mn}$ moments in the system 
and $\chi(\mathbf{q})$ is the susceptibility of the alloy. $G$ is an exchange constant discussed below; its independence from $q$ corresponds to the case of localized Mn spins. Numerical calculations were carried out for $\mathrm{Mn}$ concentrations ranging from $0.1 \%$ to $5 \%$, utilizing a spin-flipping process to arrive at ground states corresponding to different forms of $\chi(\mathbf{q})$. As in the other models, AFM states were not found when the susceptibility corresponds to the usual RKKY form, that is, for monotonically decreasing $\chi(\mathbf{q})$ (see Fig. 5). However, when a peak in $\chi(\mathbf{q})$ is put in "by hand" at $q=2 k_{F}$, the spin-flipping process resulted in AFM states at all of the concentrations studied. This result, in parallel with Overhauser's original considerations, ${ }^{6}$ provides strong evidence that ordered antiferromagnetism in CuMn alloys is stabilized by a peak in $\chi(q)$ at $2 k_{F}$. In addition to the existence of an incornmensurate AFM state, another result from Ref. 4 is that peak widths in the Fourier transform of the AFM spin structure follow the width of the peak in $\chi(\mathbf{q})$. In other words, in this model the peak width measured in a neutron-scattering measurement serves as a direct probe of the width of the peak in $\chi(\mathbf{q})$, an intrinsic property of the CuMn alloy.

In a SDW AFM model of CuMn, one begins by considering the ground state of pure copper. This could be a stable SDW state, but experimental verification of such a state is extremely difficult due to the vanishing form factor discussed above. ${ }^{16} \mathrm{Next}$, consider the case where SDWs are stabilized by the dilute addition of magnetic ions. This is possible due to the energy gain which is obtained by aligning the solute ions with the local polarization of the SDW, via the s-d exchange between the $3 \mathrm{~d}$ Mn electrons and the $4 \mathrm{~s}$ copper conduction band. Apart from the neutron-scattering form factors discussed above, localization of the magnetic solute orbitals is necessary in order for the SDW phase to be stabilized, since an orbital which extends over an entire oscillation of a SDW has no preferred orientation. ${ }^{17}$ Quantitatively, the interaction energy of a magnetic impurity and a SDW is $\mathcal{H}_{j}=-G(q) \mathbf{S}_{\mathbf{j}} \cdot \hat{\epsilon} P_{0} \cos \left(\mathbf{q} \cdot \mathbf{R}_{\mathbf{j}}\right)$, where $G(q)$ is the exchange constant between isolated impurity spins $\mathbf{S}_{\mathbf{j}}$ and a SDW with wavevector $q$ and polarization $\hat{\epsilon}$. $M n$ ions are located at $\mathbf{R}_{\mathbf{j}}$, and $P_{0}$ is the fractional polarization of conduction electrons in the SDW. ${ }^{6}$ The spatial extension of the spins $\mathbf{S}_{\mathbf{j}}$ enters through $G(q)$. After adding the (positive) SDW excitation energy to $\mathcal{H}_{j}$, assuming complete alignment of the solute moments, and minimizing with respect to $P_{0}$, the net energy change due to the $\mathrm{SDW}$ is

$$
\mathcal{H}(\mathbf{q})=-\left(\frac{2 S N_{p}}{\pi n \mu_{B}}\right)^{2} G^{2}(q) \chi(\mathbf{q}),
$$

where $N_{p}$ is the concentration of solute atoms, $n$ is the electron density, and $\mu_{B}$ is the Bohr magneton. As in the simulations discussed above, ${ }^{4} \mathrm{Eq} .2$ implies that an AFM spin state is stabilized by a peak in the susceptibility at non-zero $q$. In the original predictions of SDW instabilities by Overhauser, ${ }^{6}$ this peak consists of the singularity at $2 k_{F}$ which is obtained in Hartree-Fock theory of an electron gas with Coulomb interactions. If the electron-electron interaction is completely screened, the singularity and in fact the peak in $\chi(\mathbf{q})$ vanishes (Fig. 5). However, as shown by Fajen and Vignale, ${ }^{4}$ a singularity in $\chi(\mathbf{q})$ is not necessary: AFM ordering of magnetic solute atoms occurs when the susceptibilty contains a finite maximum at a non-zero value of $\mathbf{q}$. The Fermi surface of copper is not spherical (Fig. 6) and its asymmetry leads the possibility of preferred directions for the formation of SDW domains. The neutron-scattering results show that favored directions lie near [110], in a relatively flat region of the Fermi surface. Therefore, the value of $2 k_{F}$ along [110] is the relevant one for CuMn. 


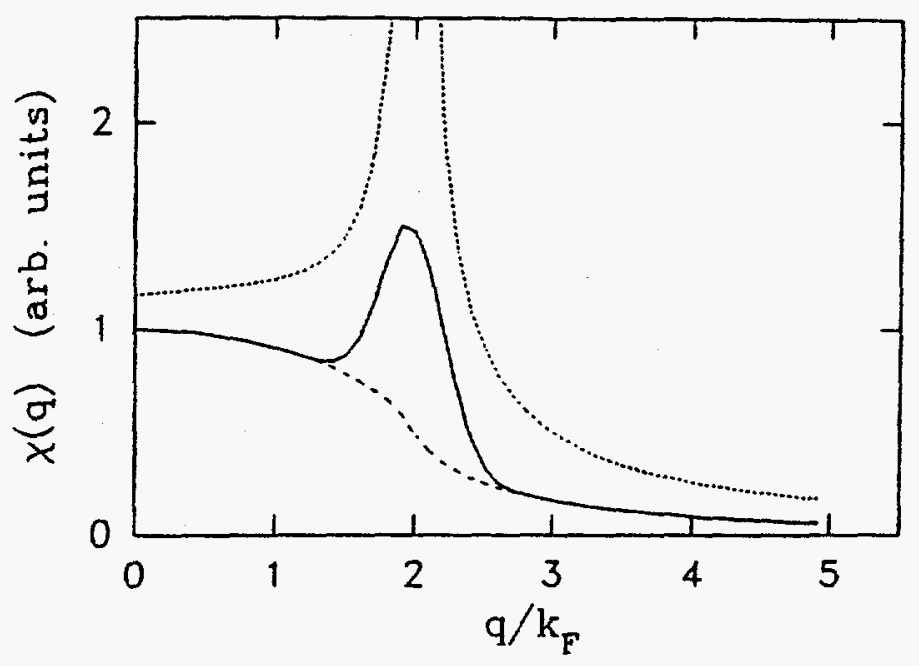

Figure 5. Schematic plot of the magrietic susceptibility $\chi(q)$, following references 4 and 6 . The three qualitative classes of behavior which we consider are a non-interacting electron gas (dashed curve), Coulomb interactions (dotted curve), and an intermediate (partially screened) case (solid curve).

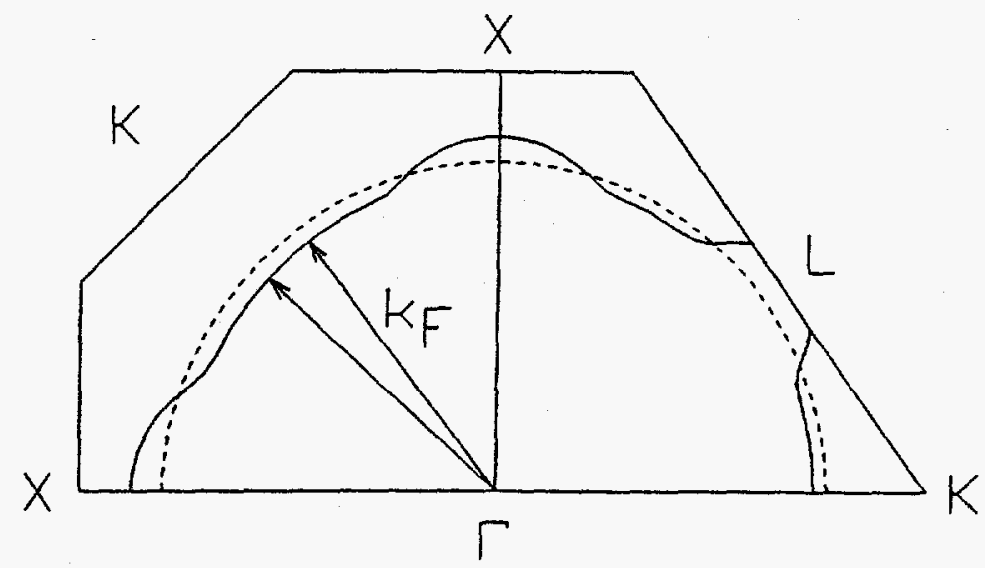

Figure 6. The Fermi surface of copper, reproduced from D. J. Roaf, Ref. 13. The arrows indicate the direction on the Fermi surface corresponding to incommensurate AFM neutron-scattering peaks in CuMn. 


\section{REFERENCES}

1. J. W. Cable, S. A. Werner, G. P. Felcher, and N. Wakabayashi, Phys. Rev. B 29, 1268 (1984).

2. Earlier neutron scattering measurements focused on clustering and relaxation phenomena. See, for example, A. P. Murani, Phys. Rev. Lett. 41, 1406 (1978).

3. L. B. Ioffe and M. V. Feigel'man, Sov. Phys. JETP 61, 354 (1985).

4. O. Fajen and G. Vignale, Solid State Comm. 77, 829 (1991).

5. K. Binder and A. P. Young, Rev. Modern Phys. 58, 801 (1986).

6. A. W. Overhauser, Phys. Rev. 128, 1437 (1962).

7. Mikhail A. Krivoglaz, Theory of X-ray and Thermal-Neutron Scattering by Real Crystals, (Plenum, New York, 1969).

8. S. C. Moss, Phys. Rev. Lett. 22, 1108 (1969).

9. J. A. Mydosh, Spin Glasses: An Experimental Introduction, (Taylor and Francis, London, 1993).

10. S. A. Werner, J. J. Rhyne, and J. A. Gotaas, Solid State Commun. 56, 457 (1985).

11. A. P. Murani and A. Heidemann, Phys. Rev. Lett. 41, 1402 (1978).

12. Y. Tsunoda, N. Kunitomi, and J. W. Cable, J. Appl. Phys. 57, 3753 (1985).

13. D. Shoenberg, Phil. Trans. A 255, 85 (1962), and D. J. Roaf, Phil. Trans. A 255, 135 (1962).

14. H. Sato, A. Arrott, and R. Kikuchi, J. Phys. Chem. Solids 10, 19 (1959).

15. J. W. Cable and Y. Tsunoda, J. Appl. Phys. 73, 5454 (1993).

16. A preliminary search for SDW features in pure $\mathrm{Cu}$ has yielded a null result. S. A. Werner, J. M. Tranquada, and G. Shirane, unpublished.

17. This was originally pointed out by A. W. Overhauser, J. Phys. Chem. Solids 13, 71 (1960).

18. Y. Tsunoda and J. W. Cable, Phys. Rev. B 46, 930 (1992).

19. F. J. Lamelas, S. A. Werner, S. M. Shapiro, and J. A. Mydosh, Phys. Rev. B (to be published, January 1, 1995).

20. An exception with rather sharp diffraction peaks is found in Ref. 18, consisting of a highly concentrated $(35 \% \mathrm{Mn})$ sample which was subjected to prolonged low-temperature anneals in order to develop ASRO. This sample clearly belongs in a different class than the typical CuMn alloys. Interestingly, SDW peaks with the usual width show up in the inelastic scans on this sample.

21. Eric Fawcett, Rev. Modern Phys. 60, 209 (1988).

22. K. Koga, K. Oshima, and N. Niimura, J. Appl. Phys. 73, 5457 (1993).

23. Samuel A. Werner, Comments Cond. Mat. Phys. 15, 55 (1990). 